\title{
Antitumor effects of heparin-polyethyleneimine nanogels delivering claudin-3-targeted short hairpin RNA combined with low-dose cisplatin on ovarian cancer
}

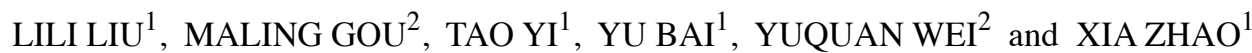 \\ ${ }^{1}$ Department of Gynecology and Obstetrics, Key Laboratory of Obstetric and Gynecologic and Pediatric Diseases \\ and Birth Defects of the Ministry of Education, West China Second Hospital, Sichuan University, \\ Chengdu, Sichuan 610041; ${ }^{2}$ State Key Laboratory of Biotherapy and Cancer Center, \\ West China Hospital, Sichuan University, Chengdu, Sichuan 610041, P.R. China
}

Received December 6, 2013; Accepted January 7, 2014

DOI: 10.3892/or.2014.2995

\begin{abstract}
Cisplatin is normally administered in chemotherapy for ovarian cancer, but is accompanied by severe dosedependent toxicity. The combination of cisplatin with other antitumor agents may be a useful alternative for achieving higher antitumor efficiency and lower toxicity. Claudin-3 (CLDN3), a commonly upregulated gene in $90 \%$ of ovarian cancers, has been identified as a novel therapeutic target of ovarian cancer. Therefore, in the present study, we constructed a recombinant plasmid carrying an shRNA targeting CLDN3 (pshCLDN3), and investigated the antitumor effects of the combination therapy of pshCLDN3 and a low-dose of cisplatin for the treatment of ovarian cancer. Heparin-polyethyleneimine (HPEI) nanogel, a novel gene carrier with superior biodegradability, excellent blood compatibility and low-toxicity, was used to deliver pshCLDN3 into ovarian cancer cells. The knockdown efficiency was determined by western blot analysis and CLDN3 immunostaining. Nude mice bearing intraperitoneal ovarian carcinomas were treated with pshCLDN3/ HPEI complexes, low-dose cisplatin, pshCLDN3/HPEI plus low-dose cisplatin or control agents, respectively. The results showed that pshCLDN3/HPEI effectively suppressed the expression of CLDN3 in ovarian cancer. The combination therapy of pshCLDN3/HPEI and low-dose cisplatin exhibited enhanced antitumor activity, when compared with either agent alone, as evidenced by mean tumor weight analysis, Ki-67 immunostaining analysis and TUNEL assay, without obvious
\end{abstract}

Correspondence to: Professor Xia Zhao, Department of Gynecology and Obstetrics, Key Laboratory of Obstetric and Gynecologic and Pediatric Diseases and Birth Defects of the Ministry of Education, West China Second Hospital, Sichuan University, No. 20, Section 3, South People's Road, Chengdu, Sichuan 610041 P.R. China

E-mail: xia-zhao@126.com

Key words: claudin-3, heparin-polyethyleneimine, short hairpin RNA, cisplatin, combination therapy, ovarian cancer systemic toxicity. These results indicate that pshCLDN3/HPEI combined with low-dose cisplatin demonstrates apparent synergistic antitumor activity without marked toxicity. Our study offers a novel therapeutic strategy for the treatment of ovarian cancer.

\section{Introduction}

Ovarian cancer remains the leading cause of death among gynecologic malignancies and accounts for $6 \%$ of all cancer-related deaths in females in the US (1). Cisplatin, as a first-line agent of cytotoxic chemotherapy for ovarian cancer is widely used in clinical practice. However, severe dosedependent toxicity, such as nephrotoxicity, neurotoxicity and ototoxicity, often cause patient intolerance $(2,3)$. Achieving the highest antitumor efficiency using the lowest possible dose is a challenging problem in the clinic. A strategy using dual agents rather than a single agent may have enormous potential for solving this issue.

Claudins (CLDNs) are a family of $17-$ to $27-\mathrm{kDa}$ integral membrane proteins forming tight junctions (TJs) (4-6). Previous studies have revealed that claudin-3 (CLDN3) and claudin-4 (CLDN4) are overexpressed in ovarian cancer (7-11) and their abnormally high expression enhances tumor cell motility, invasion and survival (12). Conversely, in vitro siRNA inhibition of CLDN3 and CLDN4 expression in ovarian cancer cells was found to reduce tumor cell invasion (12). An in vivo study carried out in our laboratory showed that silencing of the CLDN3 gene with short hairpin RNA (shRNA) significantly inhibited the growth of ovarian tumors (13). These findings suggest the importance of CLDN3 and CLDN4 as novel therapeutic targets of ovarian cancer. Furthermore, chemotherapy-resistant ovarian tumors were reported to express CLDN3 and CLDN4 genes at significantly higher levels when compared with chemotherapy-sensitive ovarian tumors (14). Thus, downregulation of CLDN4 expression may increase the chemosensitivity of ovarian cells to cisplatin (15). As a result, in the present study, we combined CLDN3 suppression with a low-dose of cisplatin for the treatment of ovarian cancer, to investigate whether there is a synergistic effect between 
CLDN3 suppression and cisplatin, and to develop a low toxic and novel therapeutic strategy against ovarian cancer.

The gene delivery system is the crucial factor that influences the efficiency of CLDN3 suppression. Traditional cationic polyethyleneimine (PEI) has become one of the most efficient non-viral gene carriers (16-18). Yet, it is not biodegradable. Moreover, its transfection efficiency is strongly correlated to its cytotoxicity, and both efficiency and cytotoxicity increase when the chain length of PEI increases $(19,20)$. In order to overcome these issues, we coupled short PEI chains into longer ones using heparin, resulting in biodegradable heparinpolyethyleneimine (HPEI) nanogels which have been proven to be efficient and low toxic in our previous studies (21-23).

In the present study, we used HPEI nanogels to deliver the plasmid expressing shRNA targeting CLDN3 (pshCLDN3) into SKOV3 human ovarian cancer cells to reduce the expression of CLDN3. Moreover, we evaluated the antitumor effects of the combination therapy of pshCLDN3/HPEI complexes and low-dose cisplatin in the treatment of ovarian cancer. Our results showed that the combination therapy exhibited an enhanced antitumor efficacy, compared with either agent alone, without obvious systemic toxicity.

\section{Materials and methods}

Plasmid vector construction. As described in our previous study (13), shRNA primers targeting CLDN3 (sense, 5'-TCCCGCAACATCATCACGTCGCATTCAAGACGTGC GACGTGATGTGTTGCTTTTTTG-3' and antisense, 5'-AGC TCAAAAAAGCAACATCATCACGTCGCACGTCTTGAA TGCGACGTGATGATGTTGC-3') were designed and synthesized. We used the HK sequence which has no homology with any of the known mammalian gene sequences as the negative control. The above sequences were then transferred into the pGenesil-2.1 vector (Genesil, Wuhan, China), which contains a kanamycin-resistance gene. The resulting recombinant plasmids were named pshCLDN3 or pshHK, respectively. Both of the two constructs were validated by DNA sequencing. The plasmids were purified using the EndoFree Plasmid Giga kit (Qiagen, Chatsworth, CA, USA) from DH5 $\alpha$ Escherichia coli transformants.

Cell culture. The human ovarian serous cystadenocarcinoma cell line SKOV3 (ATCC, Manassas, VA, USA) was cultured in Dulbecco's modified Eagle's medium (DMEM) supplemented with $10 \%$ fetal bovine serum (FBS) and antibiotics (100 units $/ \mathrm{ml}$ penicillin and $100 \mu \mathrm{g} / \mathrm{ml}$ streptomycin) in a humidified atmosphere containing $5 \% \mathrm{CO}_{2}$ at $37^{\circ} \mathrm{C}$.

Plasmid transfection. Plasmid transfection was carried out using HPEI nanogels synthesized at the State Key Laboratory of Biotherapy and Cancer Center as previously described (21). Briefly, SKOV3 cells ( $2 \times 10^{5} /$ well) were seeded in 6-well plates one day prior to transfection to achieve $80 \%$ confluence at the time of transfection. Plasmid (pshCLDN3 or pshHK)/HPEI complexes $(1 \mu \mathrm{g}$ plasmid/10 $\mu \mathrm{g}$ HPEI) were prepared in $1 \mathrm{ml}$ DMEM without serum and antibiotics. An equal volume of normal saline (NS) prepared in $1 \mathrm{ml}$ DMEM was used as a control agent. Cells were incubated with NS, HPEI nanogels, pshHK/HPEI complexes or pshCLDN3/HPEI complexes for
$6 \mathrm{~h}$, and then the medium was replaced with $2 \mathrm{ml}$ complete medium (DMEM containing 10\% FBS) and further incubated for $72 \mathrm{~h}$.

Western blot analysis. Cells or tumor tissue samples were lysed in RIPA lysis buffer containing proteinase inhibitor (1 mM cocktail plus $1 \mathrm{mM}$ PMSF). The supernatant was collected, and the protein concentration was quantified using Pierce BCA protein assay kit (Thermo Scientific, Rockford, IL, USA). Equal amounts of protein $(20 \mu \mathrm{g})$ were loaded onto $12 \%$ SDS-PAGE gel for electrophoresis and blotted onto a PVDF membrane (Millipore, Bedford, MA, USA). Subsequently, the membrane was blocked in 5\% skimmed milk for $2 \mathrm{~h}$, and then incubated with rabbit anti-human polyclonal antibody against CLDN3 (1:200; Invitrogen) at $4^{\circ} \mathrm{C}$ overnight, followed by horseradish peroxidase-conjugated secondary antibody. The immunoreactive bands were visualized by chemiluminescence detection. GAPDH served as the protein loading control.

Intraperitoneal carcinomatosis model. The following procedures for the animal experiments were approved by the Institutional Animal Care and Use Committee of Sichuan University. Pathogen-free female athymic BALB/c nude mice, 6-8 weeks of age, were used to establish the intraperitoneal carcinomatosis model as previously described (24).

Briefly, SKOV3 cells $\left(5 \times 10^{6}\right)$ in $0.1 \mathrm{ml}$ serum-free DMEM were injected s.c. into the right flank of 5 mice. Tumors were collected and minced into small particles (diameter $\leq 1 \mathrm{~mm}$ ), when the tumor diameter reached $\sim 1 \mathrm{~cm}$. The small tumor particles were then resuspended in serum-free DMEM to reach a final volume of $15 \mathrm{ml}$. Thirty nude mice were inoculated i.p. with $0.5 \mathrm{ml}$ of the above tumor particle suspension, respectively.

Therapy studies in vivo. To explore the therapeutic efficacy of pshCLDN3/HPEI plus cisplatin, we treated the mice 7 days after inoculation. The mice were randomly divided into 6 groups (5/group), and received the following intraperitoneal (i.p.) administration: (i) $100 \mu \mathrm{l}$ normal saline (NS); (ii) $50 \mu \mathrm{g}$ HPEI nanogels in $100 \mu \mathrm{l}$ NS, every two days for 12 times; (iii) $5 \mu \mathrm{g}$ pshHK/50 $\mu \mathrm{g}$ HPEI complexes in $100 \mu \mathrm{l}$ NS every two days for 12 times; (iv) $5 \mu \mathrm{g}$ pshCLDN3/50 $\mu \mathrm{g}$ HPEI complexes in $100 \mu \mathrm{l} \mathrm{NS}$, every two days for 12 times; (v) $100 \mu \mathrm{l}$ of cisplatin, weekly for 4 times ( $3 \mathrm{mg} / \mathrm{kg}$; Qinu Pharmacy Corporation, China); (vi) $5 \mu \mathrm{g}$ pshCLDN3/50 $\mu \mathrm{g}$ HPEI complexes in $100 \mu \mathrm{l}$ NS for 12 times and $100 \mu \mathrm{l}$ of cisplatin $(3 \mathrm{mg} / \mathrm{kg}$ ) for 4 times. The dose of cisplatin used in this study was $3 \mathrm{mg} / \mathrm{kg}$, since it was previously reported that treatment with $3 \mathrm{mg} / \mathrm{kg}$ cisplatin was well tolerated, and did not lead to a complete response but significantly inhibited tumor growth in an ovarian cancer model $(25,26)$.

Two days after the last intraperitoneal injection, mice were sacrificed, and the intraperitoneal tumors were collected and weighed. At the same time following sacrifice, the location of the macroscopic tumors of each mouse was carefully observed and recorded. Tumors were divided into two parts. One was fixed in $10 \%$ formalin ( $\mathrm{pH} 7.0)$ and embedded in paraffin. The other was stored at $-80^{\circ} \mathrm{C}$ for protein extraction.

Histological analysis. Paraffin-embedded intraperitoneal tumors were cut into 3 - to $5-\mu \mathrm{m}$ sections. Apoptosis was 
A

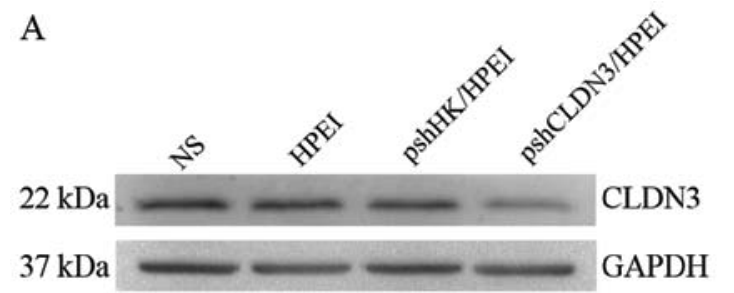

C
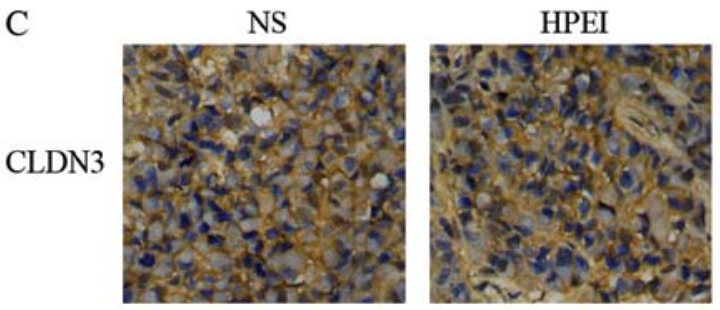

B

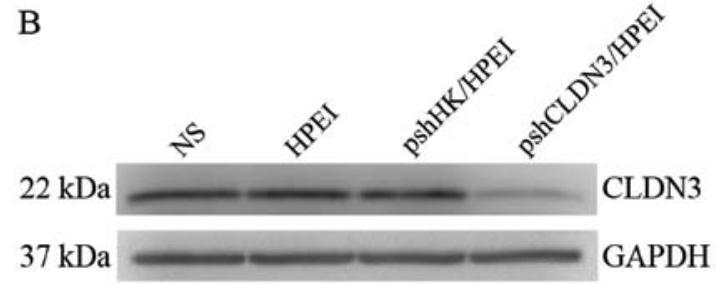

pshHK/HPEI

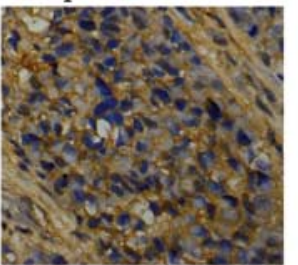

pshCLDN3/HPEI

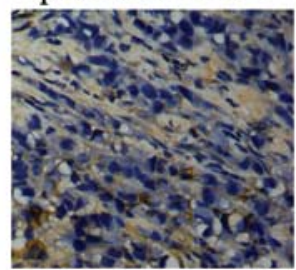

Figure 1. Downregulation of CLDN3 expression by pshCLDN3/HPEI in vitro and in vivo. (A) Western blot analysis showed a marked reduction in CLDN3 expression in the cells transfected with the pshCLDN3/HPEI complexes, when compared with that in the control groups. GAPDH was used as the internal standard. (B) Western blot analysis showed a marked reduction in CLDN3 expression in the intraperitoneal tumors after i.p. administration of the pshCLDN3/HPEI complexes. GAPDH was used as the internal standard. (C) Immunostaining of CLDN3 in tumor tissue sections (x400). Compared with the control groups, the immunostaining intensity of CLDN3 in the tumors treated with the pshCLDN3/HPEI complexes was obviously weaker. NS, normal saline; CLDN3, claudin-3; HPEI, heparin-polyethyleneimine.

evaluated by terminal deoxynucleotidyl transferase-mediated dUTP nick end labeling (TUNEL) assay according to the manufacturer's instructions (Promega, Madison, WI, USA). Cell nuclei presenting dark green fluorescence were considered to be TUNEL-positive nuclei. The apoptosis index was determined by calculating the average percentage of TUNELpositive cells in 5 random fields from 3 different sections at a magnification of $x 400$.

CLDN3 and Ki-67 immunostaining were carried out with rabbit anti-human CLDN3 antibody (1:100; Invitrogen) and rabbit anti-human Ki-67 antibody (1:100; Thermo Scientific), respectively. Briefly, tumor sections were first deparaffinized, rehydrated and incubated with $3 \% \mathrm{H}_{2} \mathrm{O}_{2}$ for $10 \mathrm{~min}$. Then, antigen retrieval was conducted using $10 \mathrm{mM}$ citrate buffer ( $\mathrm{pH}$ 8.0). After blocking for $15 \mathrm{~min}$ with normal rabbit serum, sections were incubated with the primary antibody, followed by biotinylated secondary antibody and the streptavidin-biotin complex, successively. Detection was performed using diaminobenzidine (DAB) peroxide solution, and then the cellular nuclei were counterstained with hematoxylin. The proliferation index was determined by calculating the average percentage of Ki-67-positive cells with brown-staining nuclei in 5 random fields from 3 different sections at a magnification of $x 400$.

Assessment of toxicity. Health correlated indices such as weight loss, anorexia, diarrhea, cachexia, skin ulcerations or toxic death were evaluated every 4 days to evaluate the possible side-effects. Additionally, at the termination of the animal studies, the main organs (heart, liver, spleen, lung and kidney) were fixed in $10 \%$ formalin ( $\mathrm{pH} \mathrm{7.0),} \mathrm{embedded} \mathrm{in} \mathrm{paraffin,}$ and then cut into 3- to 5- $\mu \mathrm{m}$ sections for $\mathrm{H} \& \mathrm{E}$ staining.

Statistical analysis. Values are expressed as means \pm SD. ANOVA and the Student-Newman-Keuls test were used for comparisons. $\mathrm{P}<0.05$ was considered to indicate a statistically significant result.

\section{Results}

Suppression of CLDN3 expression by pshCLDN3/HPEI complexes in SKOV3 ovarian cancer cells in vitro. Evaluation of CLDN3 expression in the SKOV3 cells was performed by western blot analysis $72 \mathrm{~h}$ after transfection. As shown in Fig. 1A, a marked reduction in CLDN3 expression was noted in the cells transfected with the pshCLDN3/HPEI complexes, whereas no apparent alteration in CLDN3 expression was observed in cells transfected with the pshHK/HPEI complexes or the HPEI nanogels, when compared with that in the blank control (NS).

Suppression of CLDN3 expression by pshCLDN3/HPEI complexes in vivo. We investigated whether pshCLDN3/HPEI complexes reduce the expression of CLDN3 in vivo. The intraperitoneal tumors were harvested for western blot analysis and immunohistochemistry at the termination of the animal experiments. Similar to the in vitro culture, the pshCLDN3/HPEI complexes markedly reduced the expression of CLDN3 (Fig. 1B), whereas the pshHK/HPEI complexes or HPEI nanogels had no discernible effect on CLDN3 expression. Similar results were observed with immunohistochemistry (Fig. 1C).

\section{Enhanced antitumor efficacy of the combination regimen} of the pshCLDN3/HPEI complexes and low-dose cisplatin. At the termination of the animal studies, the mice were sacrificed, and the location of the macroscopic tumors was carefully observed. In the NS, HPEI and pshHK/HPEI groups, each mouse developed intraperitoneally macroscopic tumor nodules scattered on various viscera. A number of tumors were observed not only deposited on the surface of the liver, but also invading the parenchyma. In the other 3 groups, the intraperitoneal tumor nodules were localized. Tumor invasion in tissues and organs was not obvious in these groups. 


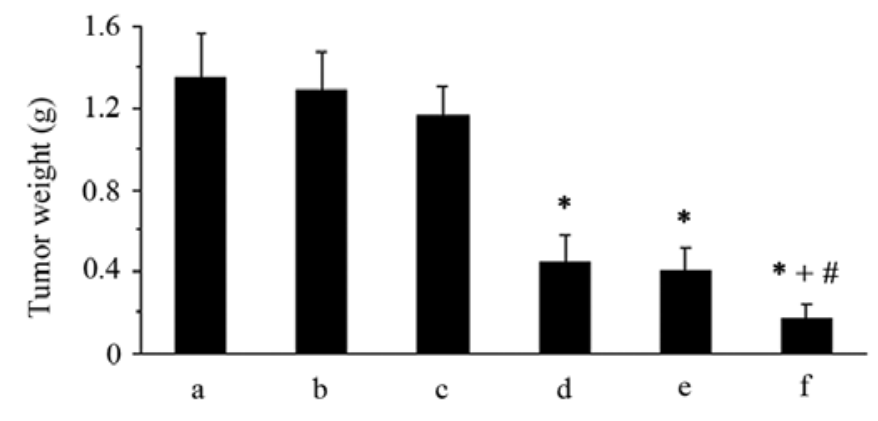

Figure 2. Tumor weights in the xenograft model of human ovarian cancer in nude mice. Nude mice bearing intraperitoneal ovarian carcinomas were divided into 6 groups (5/group), and received i.p. administration of (a) NS, (b) HPEI nanogels, (c) pshHK/HPEI complexes, (d) pshCLDN3/HPEI complexes, (e) low-dose cisplatin or (f) pshCLDN3/HPEI plus low-dose cisplatin, respectively. The results indicated that both pshCLDN3/HPEI complexes and low-dose cisplatin alone significantly inhibited the growth of ovarian carcinomas. The combination of pshCLDN3/HPEI and cisplatin displayed enhanced antitumor activity, when compared with pshCLDN3/ HPEI or cisplatin alone. Data are expressed as means $\pm \mathrm{SD}$. ${ }^{*} \mathrm{P}<0.05$ vs. the NS group; ${ }^{+} \mathrm{P}<0.05$ vs. the pshCLDN3/HPEI group; ${ }^{\#} \mathrm{P}<0.05$ vs. the cisplatin group. NS, normal saline; HPEI, heparin-polyethyleneimine; CLDN3, claudin-3.

Next, the intraperitoneal tumors were harvested and weighed. As showed in Fig. 2, the mean tumor weight was $1.35 \pm 0.21,1.29 \pm 0.18,1.16 \pm 0.14,0.45 \pm 0.13,0.40 \pm 0.11$ and $0.17 \pm 0.07 \mathrm{~g}$ in the NS, HPEI nanogel, pshHK/HPEI complex, pshCLDN3/HPEI complex, low-dose cisplatin and pshCLDN3/HPEI complex plus low-dose cisplatin group, respectively. The data showed that the pshCLDN3/HPEI complexes or low-dose cisplatin alone significantly inhibited tumor growth, compared with the control therapies $(\mathrm{P}<0.05)$. Furthermore, the combination of pshCLDN3/HPEI and cisplatin had a superior antitumor effect, compared with pshCLDN3/HPEI or cisplatin alone $(\mathrm{P}<0.05)$. No significant difference in tumor weight was found among the control groups $(\mathrm{P}>0.05)$.

Inhibition of proliferation in vivo. Ki-67 immunostaining was used to evaluate the tumor cell proliferation in each group. As shown in Fig. 3A and B, both the pshCLDN3/HPEI complexes and low-dose cisplatin monotherapy group exhibited weak staining for Ki-67, when compared with the control groups $(\mathrm{P}<0.05)$. No significant difference in $\mathrm{Ki}-67$ staining was visible in the two groups $(\mathrm{P}>0.05)$. Moreover, the percentage of Ki-67-positive cells was significantly reduced in the combination therapy group, when compared with the percentage in the pshCLDN3/HPEI or low-dose cisplatin monotherapy group $(\mathrm{P}<0.05)$.

Induction of apoptosis in vivo. We used TUNEL assay to detect apoptotic cells in the tumor tissues of each group. The results showed that both the pshCLDN3/HPEI and cisplatin monotherapy resulted in a significant increase in apoptotic tumor cells when compared with that following treatment with the control agents $(\mathrm{P}<0.05)$. Tumors in the combination therapy group showed an increased number of positive nuclei, when compared with the number in the pshCLDN3/HPEI or low-dose cisplatin monotherapy group $(\mathrm{P}<0.05)$. However, positive nuclei were rare in the control groups (Fig. 3A and C).
A
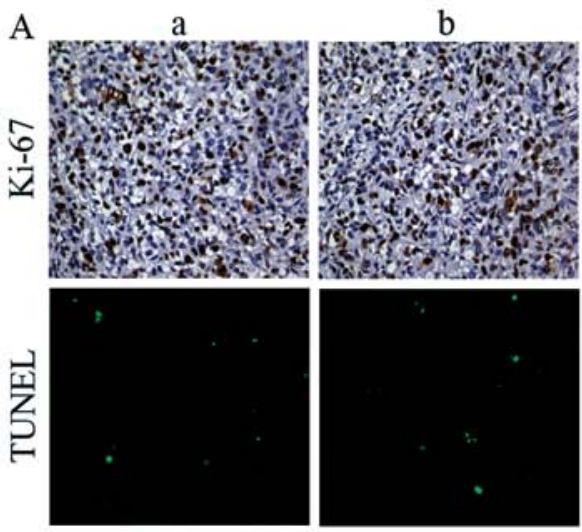

B

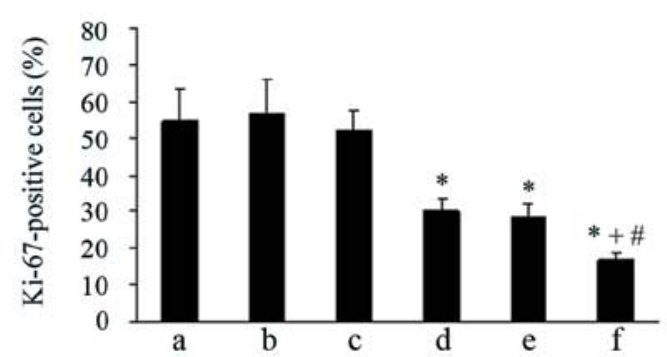

c
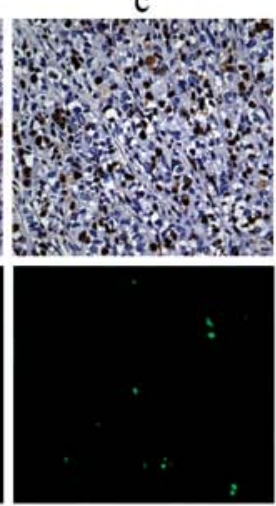
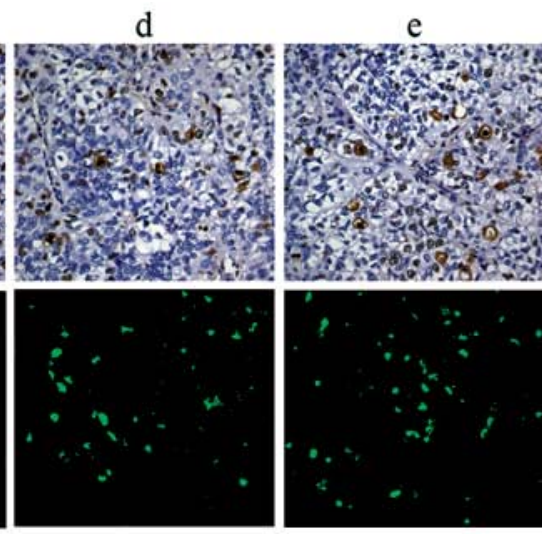

C

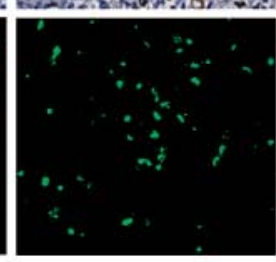

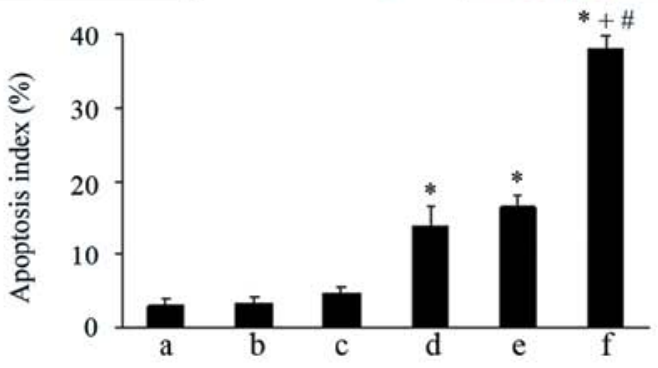

Figure 3. Ki-67 immunostaining and TUNEL assay of tumor tissues in the different groups. (A) Top panel, tumor sections immunostained with rabbit antihuman Ki-67 antibody (x200). Bottom panel, tumor sections stained for TUNEL (x200). (a-f) NS, HPEI, pshHK/HPEI, pshCLDN3/HPEI, low-dose cisplatin and pshCLDN3/HPEI plus low-dose cisplatin group, respectively. (B) Quantification of Ki-67-positive cells. (C) Quantification of TUNEL staining (apoptotic index). Data are presented as means $\pm \mathrm{SD}$. " $\mathrm{P}<0.05$ vs. the NS group; ${ }^{+} \mathrm{P}<0.05$ vs. the pshCLDN3/HPEI group; $\mathrm{P}<0.05$ vs. the cisplatin group. NS, normal saline; HPEI, heparin-polyethyleneimine; CLDN3, claudin-3. 


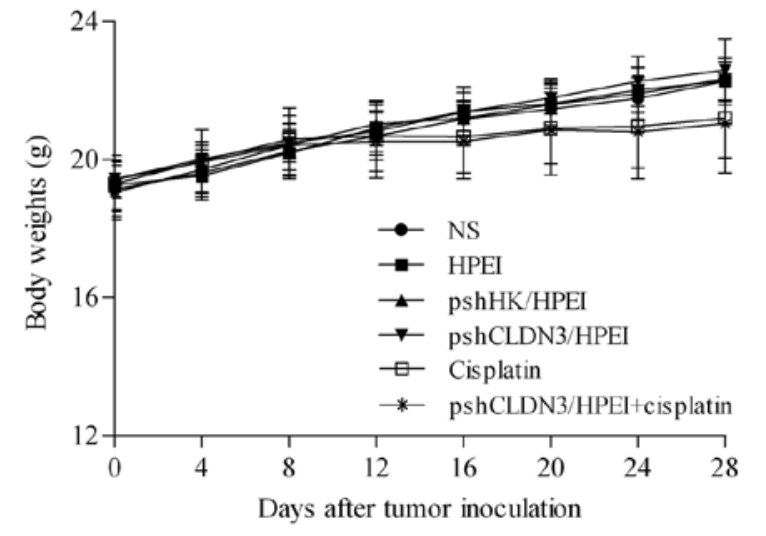

Figure 4. Body weights of the mice in each group. Mice were weighed every 4 days. Although the mean body weights in the two cisplatin-treated groups were less than that in the other 4 groups after the treatment course, the differences was not statistically significant $(\mathrm{P}>0.05)$. Data are expressed as the means \pm SD. NS, normal saline; HPEI, heparin-polyethyleneimine; CLDN3, claudin-3

Observation of toxicity. In the present study, no gross abnormalities were observed in any group. As shown in Fig. 4, although the mean body weights in the two cisplatin-treated groups were less than that in the other four groups after the treatment course, the differences did not reach statistical significance $(\mathrm{P}>0.05)$. Furthermore, no pathologic change in the heart, liver, spleen, lung or kidney was noted by microscopic examination after the administration of pshCLDN3/HPEI or pshCLDN3/HPEI plus cisplatin.

\section{Discussion}

In the present study, we demonstrated that pshCLDN3/HPEI complexes effectively inhibited ovarian tumor growth, reduced tumor cell proliferation and increased tumor cell apoptosis. In addition, from the differences in the tumor location among the groups, we inferred that pshCLDN3/HPEI complexes also inhibited ovarian tumor invasion. These findings are consistent with a previous study carried out in our laboratory, in which pshCLDN3 was delivered by polyp(lactic-co-glycolic acid) (PLGA) (13), and another study carried out by Huang et al (27). Moreover, in the present study, the combination therapy of pshCLDN3/HPEI and low-dose cisplatin exhibited enhanced antitumor activity, compared with either agent alone, as evidenced by analysis of the mean tumor weight, Ki-67 immunostaining analysis and TUNEL assay, indicating that there may be a synergistic effect between CLDN3 suppression and cisplatin in the treatment of ovarian cancer. The combination therapy holds much promise as an effective strategy against ovarian cancer.

In addition to efficacy, the safety of therapy is an important factor in considering its utility for clinical application. It has been reported that CLDN3 is also expressed in several other normal organs and tissues $(28,29)$. Systemic administration of pshCLDN3/HPEI may interfere with the expression of CLDN3 in these tissues, thus, causing various unpredictable side-effects. Therefore, in the present study, we utilized the i.p. route for pshCLDN3/HPEI administration, which may reduce the adverse effects of silencing CLDN3 in normal tissues that reside outside the peritoneum. Moreover, considering the severe side-effects owing to a high dose of cisplatin, we used a low-dose of cisplatin $(3 \mathrm{mg} / \mathrm{kg})$ in this study. Although the final mean body weights in the two cisplatin-treated groups were less than that in the other four groups, the differences did not reach statistical significance $(\mathrm{P}>0.05)$. Overall, the i.p. administration of pshCLDN3/HPEI combined with lowdose cisplatin was well tolerated, with no obvious toxicity throughout the course of treatment, and macroscopic examination of vital organs was normal at sacrifice. These results indicate that the combination therapy of pshCLDN3/HPEI and low-dose cisplatin has low toxicity and has potential clinical application.

However, to date, the exact mechanisms of how the combination therapy exerts its effect remain unclear. Two possible mechanisms may be involved. First, the enhanced antitumor effect may result from the enhanced activity of induced apoptosis. In the present study, we observed a higher number of apoptotic cells in the tumors treated with pshCLDN3/HPEI plus cisplatin, compared with the pshCLDN3/HPEI or cisplatin alone group (Fig. 3A and C). Cisplatin is a well-known DNA damaging agent. It has the capability to form platinum-DNA adducts which activate several cellular processes, ultimately leading to cell cycle arrest, transcription inhibition and cell apoptosis (30-32). Suppression of CLDN3 in ovarian cancer cells has also been found to induce cell apoptosis $(13,27)$. Thus, there may be a common synergistic apoptotic pathway between pshCLDN3/HPEI and cisplatin. Second, the enhanced antitumor effect may result from the increased penetration of cisplatin into tumor tissues. Tight junctions (TJs) act as a barrier and regulator of the passage of molecules and ions between cells. CLDNs, as the major component of TJs, influence barrier functions. It has been suggested that CLDN suppression in tumor cells increases TJ permeability, thus, increasing the penetration of chemotherapeutic agents into tumor tissues, resulting in greater effectiveness of the chemotherapeutic agents $(15,33)$. Therefore, we infer that pshCLDN3/HPEI increases the penetration of cisplatin into ovarian tumor tissues, and consequently enhances the antitumor effect of cisplatin. Moreover, CLDNs are transmembrane proteins and are associated with the membrane permeability of molecules. A recent study using fluorescence-labeled cisplatin showed that downregulation of CLDN4 expression in ovarian cancer cells in vitro resulted in an increased cellular accumulation of fluorescence-labeled cisplatin, indicating that CLDN suppression may also affect the transmembrane transportation of cisplatin, and consequently enhance its antitumor effect (15). However, the precise mechanisms by which pshCLDN3/HPEI plus cisplatin exerts its effect require further investigation.

The application of gene therapy in cancer treatment depends on a safe and efficient gene delivery system. Although viral carriers have high transfection efficiency, they consistently cause severe side-effects (34). In contrast, non-viral gene carriers such as cationic lipids and cationic polymers have many advantages, including the ease of production, low immunogenicity, and feasibility of delivering larger DNA molecules $(35,36)$. However, toxicity also hinders their applications (37). In our previous studies, we developed a novel non-viral gene delivery system using HPEI nanogels. Different from traditional PEI, HPEI nanogels are biodegrad- 
able. Moreover, they are less toxic, and have a better blood compatibility $(21,22)$. Based on these advantages, we used HPEI nanogels as the gene carrier in our present study. Our data showed that pshCLDN3/HPEI complexes significantly reduced the expression of CLDN3 in vitro and in vivo, indicating that HPEI nanogels efficiently deliver pshCLDN3 into SKOV3 human ovarian cancer cells. No apparent cytotoxicity and systemic toxic effects of HPEI nanogels were found in this study. pshCLDN3 delivered by HPEI nanogels showed an excellent tolerance throughout the treatment process.

In conclusion, our data showed that pshCLDN3/HPEI complexes obviously inhibited the growth of ovarian cancer. The combination therapy of pshCLDN3/HPEI and low-dose cisplatin exhibited enhanced antitumor activity, when compared with either agent alone, without obvious toxicity. The HPEI nanogel as a new non-viral gene carrier exhibited high efficiency and low toxicity. Our study offers a novel and promising therapeutic strategy for human ovarian cancer.

\section{Acknowledgements}

This study was supported by the National 973 Program of China (2010CB529905, 2011CB910703), the National Natural Science Foundation of China (NSFC81071861), the Specialized Research Fund for the Docoral Program of Higher Education of China (20120181110029), and the National Science and Technology Major Project (2009zx09503-020).

\section{References}

1. Siegel R, Naishadham D and Jemal A: Cancer statistics, 2012. CA Cancer J Clin 62: 10-29, 2012.

2. McKeage MJ: Comparative adverse effect profiles of platinum drugs. Drug Saf 13: 228-244, 1995.

3. Piccart MJ, Lamb H and Vermorken JB: Current and future potential roles of the platinum drugs in the treatment of ovarian cancer. Ann Oncol 12: 1195-1203, 2001.

4. Morita K, Furuse M, Fujimoto K and Tsukita S: Claudin multigene family encoding four-transmembrane domain protein components of tight junction strands. Proc Natl Acad Sci USA 96: 511-516, 1999.

5. Tsukita $\mathrm{S}$ and Furuse M: Pores in the wall: claudins constitute tight junction strands containing aqueous pores. J Cell Biol 149: $13-16,2000$.

6. Tsukita S, Furuse M and Itoh M: Multifunctional strands in tight junctions. Nat Rev Mol Cell Biol 2: 285-293, 2001.

7. Hough CD, Sherman-Baust CA, Pizer ES, et al: Large-scale serial analysis of gene expression reveals genes differentially expressed in ovarian cancer. Cancer Res 60: 6281-6287, 2000

8. Rangel LB, Agarwal R, D'Souza T, et al: Tight junction proteins claudin-3 and claudin- 4 are frequently overexpressed in ovarian cancer but not in ovarian cystadenomas. Clin Cancer Res 9: 2567-2575, 2003.

9. Hibbs K, Skubitz KM, Pambuccian SE, et al: Differential gene expression in ovarian carcinoma: identification of potential biomarkers. Am J Pathol 165: 397-414, 2004.

10. Lu KH, Patterson AP, Wang L, et al: Selection of potential markers for epithelial ovarian cancer with gene expression arrays and recursive descent partition analysis. Clin Cancer Res 10: 3291-3300, 2004.

11. Santin AD, Zhan F, Bellone S, et al: Gene expression profiles in primary ovarian serous papillary tumors and normal ovarian epithelium: identification of candidate molecular markers for ovarian cancer diagnosis and therapy. Int J Cancer 112: 14-25, 2004.

12. Agarwal R, D'Souza T and Morin PJ: Claudin-3 and claudin-4 expression in ovarian epithelial cells enhances invasion and is associated with increased matrix metalloproteinase- 2 activity. Cancer Res 65: 7378-7385, 2005.

13. Sun C, Yi T, Song X, et al: Efficient inhibition of ovarian cancer by short hairpin RNA targeting claudin-3. Oncol Rep 26 193-200, 2011
14. Santin AD, Cané S, Bellone S, et al: Treatment of chemotherapyresistant human ovarian cancer xenografts in C.B-17/SCID mice by intraperitoneal administration of Clostridium perfringens enterotoxin. Cancer Res 65: 4334-4342, 2005.

15. Yoshida H, Sumi T, Zhi X, Yasui T, Honda K and Ishiko O: Claudin-4: a potential therapeutic target in chemotherapy-resistant ovarian cancer. Anticancer Res 31: 1271-1277, 2011.

16. Boussif O, Lezoualc'h F, Zanta MA, et al: A versatile vector for gene and oligonucleotide transfer into cells in culture and in vivo: polyethylenimine. Proc Natl Acad Sci USA 92: 7297-7301, 1995.

17. Lungwitz $U$, Breunig $M$, Blunk $T$ and Göpferich $A$ : Polyethylenimine-based non-viral gene delivery systems. Eur J Pharm Biopharm 60: 247-266, 2005.

18. Neu M, Fischer D and Kissel T: Recent advances in rational gene transfer vector design based on poly(ethylene imine) and its derivatives. J Gene Med 7: 992-1009, 2005.

19. Godbey WT, Wu KK and Mikos AG: Size matters: molecular weight affects the efficiency of poly(ethylenimine) as a gene delivery vehicle. J Biomed Mater Res 45: 268-275, 1999.

20. Kunath K, von Harpe A, Fischer D, et al: Low-molecular-weight polyethylenimine as a non-viral vector for DNA delivery: comparison of physicochemical properties, transfection efficiency and in vivo distribution with high-molecular-weight polyethylenimine. J Control Release 89: 113-125, 2003.

21. Gou M, Men K, Zhang J, et al: Efficient inhibition of C-26 colon carcinoma by VSVMP gene delivered by biodegradable cationic nanogel derived from polyethyleneimine. ACS Nano 4: 5573-5584, 2010.

22. Xie C, Gou ML, Yi T, et al: Efficient inhibition of ovarian cancer by truncation mutant of FILIP1L gene delivered by novel biodegradable cationic heparin-polyethyleneimine nanogels. Hum Gene Ther 22: 1413-1422, 2011.

23. Liu P, Gou M, Yi T, et al: The enhanced antitumor effects of biodegradable cationic heparin-polyethyleneimine nanogels delivering HSulf-1 gene combined with cisplatin on ovarian cancer. Int J Oncol 41: 1504-1512, 2012.

24. Lin XJ, Chen XC, Wang L, et al: Dynamic progression of an intraperitoneal xenograft model of human ovarian cancer and its potential for preclinical trials. J Exp Clin Cancer Res 26: 467-474, 2007.

25. Mabuchi S, Altomare DA, Cheung M, et al: RAD001 inhibits human ovarian cancer cell proliferation, enhances cisplatininduced apoptosis, and prolongs survival in an ovarian cancer model. Clin Cancer Res 13: 4261-4270, 2007.

26. Mabuchi S, Terai Y, Morishige K, et al: Maintenance treatment with bevacizumab prolongs survival in an in vivo ovarian cancer model. Clin Cancer Res 14: 7781-7789, 2008.

27. Huang YH, Bao Y, Peng W, et al: Claudin-3 gene silencing with siRNA suppresses ovarian tumor growth and metastasis. Proc Natl Acad Sci USA 106: 3426-3430, 2009.

28. Kiuchi-Saishin Y, Gotoh S, Furuse M, Takasuga A, Tano Y and Tsukita S: Differential expression patterns of claudins, tight junction membrane proteins, in mouse nephron segments. J Am Soc Nephrol 13: 875-886, 2002.

29. Hewitt KJ, Agarwal R and Morin PJ: The claudin gene family: expression in normal and neoplastic tissues. BMC Cancer 6: 186, 2006.

30. Vaisman A, Varchenko M, Said I and Chaney SG: Cell cycle changes associated with formation of Pt-DNA adducts in human ovarian carcinoma cells with different cisplatin sensitivity. Cytometry 27: 54-64, 1997.

31. Cohen SM and Lippard SJ: Cisplatin: from DNA damage to cancer chemotherapy. Prog Nucleic Acid Res Mol Biol 67: 93-130, 2001.

32. Wang D and Lippard SJ: Cellular processing of platinum anticancer drugs. Nat Rev Drug Discov 4: 307-320, 2005.

33. Kominsky SL: Claudins: emerging targets for cancer therapy. Expert Rev Mol Med 8: 1-11, 2006.

34. Relph K, Harrington $\mathrm{K}$ and Pandha H: Recent developments and current status of gene therapy using viral vectors in the United Kingdom. BMJ 329: 839-842, 2004.

35. Glover DJ, Lipps HJ and Jans DA: Towards safe, non-viral therapeutic gene expression in humans. Nat Rev Genet 6: 299-310, 2005.

36. Ferber D: Gene therapy. Safer and virus-free? Science 294: 1638-1642, 2001.

37. Lv H,Zhang S, Wang B, Cui S and Yan J: Toxicity of cationic lipids and cationic polymers in gene delivery. J Control Release 114: 100-109, 2006. 